\title{
AN ORTHOMODULAR LATTICE ADMITTING NO GROUP-VALUED MEASURE
}

\author{
MIRKO NAVARA
}

(Communicated by Jeffry N. Kahn)

\begin{abstract}
We construct a finite orthomodular lattice $L$ such that, for each commutative group $G$, there is no nontrivial $G$-valued measure on $L$. This result extends a result of $\mathbf{R}$. J. Greechie (Orthogonal lattices admitting no states, J. Combin. Theory Ser. A 10 (1971), 119-132), and also sheds light on recent investigations in the noncommutative measure theory.
\end{abstract}

\section{INTRODUCTION}

In the quantum logic approach to the foundations of quantum mechanics it is often assumed that the "event structure" of a quantum experiment is an orthomodular lattice (OML) $L$ (see [7, 16, 18], etc.). The "states" of the experiment are then represented by probability measures on $L$. Thus, it is natural in this general approach to ask if any OML admits probability measures, as all Boolean OMLs and Hilbert-space-projector OMLs do, or if there are OMLs with only very few or no probability measures. This question was answered by Greechie-he showed that there are finite OMLs without any probability measure at all [5]. As peculiar as the latter result might seem, it considerably influenced the quantum axiomatics (see [7, 16] - the existence of "states" has to be assumed!) and both algebraic and analytic investigations of OMLs (see $[11,14,17]$, etc.).

In a generalized setup of vector-valued or group-valued measures on OMLs, an analogous question has apparently been an open problem (the Greechie examples [5] as well as the other combinatorial examples [15, 16, 17] do admit nontrivial group-valued measures). Since quite a few Boolean group-valued measure-theoretic results have found their noncommutative versions recently (such as, e.g., the generalization of [1] and [19] established in [4]), this question of the existence of group-valued measures on an OML has become even more topical. In this paper we provide an answer to the question by exhibiting a finite OML with no nontrivial group-valued measure. In the proof we have adopted the basic Greechie construction idea, but we also needed to combine it with some new combinatorial methods in OMLs.

Received by the editors April 30, 1992 and, in revised form, November 17, 1992.

1991 Mathematics Subject Classification. Primary 06C15, 28B10; Secondary 03G12.

Key words and phrases. Orthomodular lattice, group-valued measure, Greechie diagram. 


\section{Preliminaries}

Let us first review basic notions as we shall use them in the sequel.

An orthomodular lattice is a lattice $L$ with a least and a greatest element (denoted by 0,1 , respectively) and with a unary operation ' (orthocomplementation) satisfying the following properties for all $a, b \in L$ :

(1) $\left(a^{\prime}\right)^{\prime}=a$,

(2) $a \leq b \Longrightarrow b^{\prime} \leq a^{\prime}$,

(3) $a \vee a^{\prime}=1$,

(4) $a \leq b \Longrightarrow b=a \vee\left(b \wedge a^{\prime}\right)$.

Let $L$ be an orthomodular lattice, and let $G$ be a commutative additive group. A mapping $m: L \rightarrow G$ is called a $G$-valued measure on $L$ if the following conditions are satisfied:

(1) $m(0)=0$,

(2) $m(a \vee b)=m(a)+m(b)$ whenever $a, b$ are orthogonal, i.e., $a \leq b^{\prime}$.

Obviously, every OML $L$ admits a trivial group-valued measure $m$ satisfying $m(a)=0$ for all $a \in L$. The question appears if, for a given $L$, there exists a group $G$ such that $L$ admits a nontrivial $G$-valued measure (naturally, if $L$ is a Boolean algebra then the latter question answers positively for any $G)$. As a matter of fact, all explicitly known examples of OMLs without probability measures (see $[5,15,16]$ ) admit nontrivial $Z$-valued measures (where $Z$ is the group of all integers). The following simple properties of group-valued measures will be utilized later on.

Proposition 2.1. Let $L$ be an $O M L$, and let $G$ be the group-theoretic product of commutative groups $G_{i}, i \in I$. For all $i \in I$, let $\pi_{i}: G \rightarrow G_{i}$ be the canonical projection. If $m$ is a G-valued measure on $L$, then $\pi_{i} \circ m$ is $a G_{i}$-valued measure on $L$.

The proof is elementary.

Corollary 2.2. Let $L$ be a finite OML which admits a nontrivial group-valued measure. Then there is a prime number $p$ such that $L$ admits a nontrivial $Z_{p}$-valued measure ( $Z_{p}$ is the cyclic group with $p$ elements).

Proof. Let $m$ be a nontrivial group-valued measure on $L$. The values of $m$ generate a subgroup, $\bar{G}$, of $G$. As $\bar{G}$ is finitely generated, it is isomorphic to some finite product $\prod_{i \in I} G_{i}$, where each $G_{i}$ is either $Z$ or $Z_{p^{k}}$ for some prime number $p$ and $k \in N[10, \S 20]$. In view of Proposition $2.1, L$ admits a nontrivial measure, $\bar{m}$, with values either in $Z$ or $Z_{p^{k}}$. In the former case, it suffices to factorize $Z$ modulo $q$, where $q$ is a sufficiently large prime number. If $h_{q}$ is the factorization mapping, then $h_{q} \circ \bar{m}$ is a nontrivial $Z_{q^{-}}$ valued measure. In the latter case, we factorize $Z_{p^{k}}$ modulo $p$. Again, we denote by $h_{p}$ the corresponding mapping. The measure $h_{p} \circ \bar{m}$ is nontrivial because $\bar{G}$ is generated by the values of $m$, and it is a $Z_{p}$-valued measure.

Let us now briefly recall basic technical tools of our construction. A hypergraph is a couple $H=(V, \mathscr{E})$ consisting of a nonempty set $V$ (of vertices) and a covering $\mathscr{E}$ of $V$ by nonempty subsets (edges). A loop of order $n$ in $H(n \geq 3)$ is an $n$-tuple of edges $E_{1}, \ldots, E_{n} \in \mathscr{E}$ such that the intersections 
$E_{1} \cap E_{2}, \ldots, E_{n-1} \cap E_{n}, E_{n} \cap E_{1}$ are nonempty and mutually disjoint. (Our definition of a loop slightly differs from that of [5], but this has no influence on the following Loop Lemma.)

Let $L$ be a finite OML, and let us denote by $\mathscr{A}(L)$ the set of all atoms of $L$. The Greechie diagram of $L$ (see [5]) is a hypergraph $H=(V, \mathscr{E})$ such that $V=\mathscr{A}(L)$ and $\mathscr{E}$ consists of all maximal subsets of mutually orthogonal elements of $\mathscr{A}(L)$.

Loop Lemma (see $[5,9])$. Let $H=(V, \mathscr{E})$ be a hypergraph satisfying the following conditions:

(1) $\forall E \in \mathscr{E}: \operatorname{card}(E) \geq 3$,

(2) $\forall E, F \in \mathscr{E}, E \neq F: \operatorname{card}(E \cap F) \leq 1$,

(3) there is no loop of order less than 5 in $H$.

Then there is an OML $M$ such that $H$ is the Greechie diagram of $M$.

\section{Results}

We start with an auxiliary construction (which, we believe, may find applications elsewhere, too).

Example 3.1. Let $H$ be a hypergraph whose vertices are $a_{i}, b_{i}(i=0, \ldots, 21)$ and whose edges are $\left\{a_{i}, b_{i}, a_{i+1}\right\},\left\{b_{i}, a_{i+4}, b_{i+10}\right\}(i=0, \ldots, 21)$. (Throughout this paragraph, the indices are counted modulo 22.) Applying the Loop Lemma to $H$, we obtain an OML, $M$. Notice that $M$ is invariant under any cyclic transformation of indices. (Let us remark that in the following analysis of group-valued measures on $M$ certain facts were obtained by computer-aided calculations. Nevertheless, we provide arguments which allow easy verification.)

Let $G$ be a commutative group and let $m$ be a $G$-valued measure on $M$. There is the following system of 44 linear equations for 45 variables $m(1)$, $m\left(a_{i}\right)$, and $m\left(b_{i}\right) \quad(i=0, \ldots, 21)$ :

$$
\begin{array}{ll}
\left(A_{i}\right) & m\left(a_{i}\right)+m\left(b_{i}\right)+m\left(a_{i+1}\right)=m(1) \quad(i=0, \ldots, 21), \\
\left(B_{i}\right) & m\left(b_{i}\right)+m\left(a_{i+4}\right)+m\left(b_{i+10}\right)=m(1) \quad(i=0, \ldots, 21) .
\end{array}
$$

If we combine three equations (e.g., $\left.\left(A_{0}\right),\left(B_{0}\right),\left(A_{10}\right)\right)$, we eliminate the variables $m\left(b_{i}\right)$ :

$$
m\left(a_{i}\right)+m\left(a_{i+1}\right)-m\left(a_{i+4}\right)+m\left(a_{i+10}\right)+m\left(a_{i+11}\right)=m(1)
$$

$(i=0, \ldots, 21)$. Forming the linear combination of equations $\left(C_{i}\right)$ with coefficients $(2,-13,-7,2,2,8,14,5,20,11,-4,20,20,-4,-4,14,-16,-7$, $-22,5,-28,5)$, we obtain

$$
69 m\left(a_{0}\right)=23 m(1) .
$$

By the cyclic transformation of indices, we infer that

$$
69 m\left(a_{i}\right)=23 m(1) \quad(i=0, \ldots, 21) .
$$

Thus, if $G$ is either $Z$ or a cyclic group $Z_{p}$, where $p$ is a prime number different from 3 and 23 , then $M$ admits only those $G$-valued measures which 
are constant on $\mathscr{A}(M)$. (In fact, this holds also for $Z_{3}$. However, $M$ does admit $Z_{23}$-valued measures which are not constant on $\mathscr{A}(M)$.)

Let us now explicitly state certain properties of $M$.

Lemma 3.2. Each group-valued measure $m$ on $M$ is uniquely determined by the values $m\left(a_{0}\right), m\left(a_{1}\right), m\left(a_{2}\right)$, and $m(1)$.

Proof. The linear combination of equations $\left(C_{i}\right)$ with coefficients $(-7,0,-5$, $-6,-1,3,6,8,10,10,9,6,9,12,4,0,0,-2,-12,-8,-11,-6)$ yields

$$
14 m\left(a_{0}\right)+22 m\left(a_{1}\right)+22 m\left(a_{2}\right)-m\left(a_{3}\right)=19 m(1) .
$$

This determines the value $m\left(a_{3}\right)$. Analogously, the values $m\left(a_{1}\right), m\left(a_{2}\right)$, $m\left(a_{3}\right)$, and $m(1)$ determine $m\left(a_{4}\right)$, etc. Equations $\left(A_{i}\right)$ determine the values $m\left(b_{i}\right) \quad(i=0, \ldots, 21)$.

Lemma 3.3. There is no nontrivial group-valued measure $m$ on $M$ such that $m\left(a_{6}\right)=m\left(a_{9}\right)=m\left(a_{12}\right)=m\left(a_{15}\right)=0$.

Proof. Let us form the linear combination of equations $\left(C_{i}\right)$ with coefficients $(3,12,11,1,7,11,-9,7,-9,1,-10,2,-3,-8,-10,-5,0,-6,-1,4,5,-3)$. We obtain

$$
-16 m\left(a_{6}\right)-17 m\left(a_{9}\right)+31 m\left(a_{12}\right)+m\left(a_{15}\right)+m\left(a_{18}\right)=0 .
$$

This implies $m\left(a_{18}\right)=0$. Again, the induction argument gives $0=m\left(a_{21}\right)=$ $m\left(a_{24}\right)=\cdots$. Equations $\left(A_{i}\right)$ force $m\left(b_{i}\right)=0(i=0, \ldots, 21)$.

We are now ready to formulate and prove our main result.

Theorem 3.4. There exists an OML $L$ such that, for each commutative group $G$, the only $G$-valued measure on $L$ is the trivial one.

Proof. Let us take six copies $H^{0}, \ldots, H^{5}$ of the hypergraph $H$ from Example 3.1. Let us denote their vertices by $a_{i}^{j}, b_{i}^{j}(j=0, \ldots, 5)$. Let us identify $a_{0}^{0}$ with all $a_{0}^{j}(j=1, \ldots, 5)$. Let us perform analogous identification with $a_{1}^{j}, a_{2}^{j}, b_{0}^{j}$, and $b_{1}^{j}(j=0, \ldots, 5)$. As a result of the former identification, the hypergraphs $H^{j}(j=0, \ldots, 5)$ have common vertices $a_{0}^{0}, a_{1}^{0}, a_{2}^{0}, b_{0}^{0}$, and $b_{1}^{0}$. Also, the procedure did not lead to any loop of order less than 5. Further, let us add eight "new" edges $\left\{a_{i}^{0}, a_{i}^{1}, a_{i}^{2}\right\},\left\{a_{i}^{2}, a_{i}^{3}, a_{i}^{4}, a_{i}^{5}\right\}, i=6,9,12,15$. Let us denote the resulted hypergraph by $K$. Applying the Loop Lemma to $K$, we obtain an OML $L$ associated with $K$.

Let $m$ be a group-valued measure on $L$. According to Lemma $3.2, m$ is uniquely determined by the values $m\left(a_{0}^{0}\right), m\left(a_{1}^{0}\right), m\left(a_{2}^{0}\right)$, and $m(1)\left(=m\left(a_{0}^{0}\right)+\right.$ $\left.m\left(b_{0}^{0}\right)+m\left(a_{1}^{0}\right)\right)$. Hence, $m\left(a_{i}^{j}\right)=m\left(a_{i}^{0}\right)(i=0, \ldots, 21 ; j=0, \ldots, 5)$. For the "new" edges we obtain

$$
m\left(a_{i}^{0}\right)+m\left(a_{i}^{1}\right)+m\left(a_{i}^{2}\right)=m\left(a_{i}^{2}\right)+m\left(a_{i}^{3}\right)+m\left(a_{i}^{4}\right)+m\left(a_{i}^{5}\right)=m(1)
$$

$(i=6,9,12,15)$. Using the consequences of Lemma 3.2, we see that

$$
3 m\left(a_{i}^{0}\right)=4 m\left(a_{i}^{0}\right)=m(1) \quad(i=6,9,12,15) .
$$

Thus, $m\left(a_{i}^{0}\right)=0(i=6,9,12,15)$. According to Lemma 3.3, $m$ has to be the trivial $G$-valued measure. The proof is finished.

Let us conclude with a few remarks. 
Remarks. 1. If we treat the same question for orthomodular posets, our task is substantially easier. Indeed, Greechie and Miller [6] have constructed an orthomodular poset $P$ with 22 atoms which admits exactly one probability measure. This poset $P$ may be used in place of $M$ in our construction. For every group $G$ the poset $P$ admits only those $G$-valued measures which are constant on $\mathscr{A}(P)$.

2. According to $\left(D_{i}\right)$, our lattice $M$ admits exactly one probability measure (attaining the value $1 / 3$ at each atom). Similar examples have been found in $[12,13]$. The lattice $M$ presented here, however, is considerably smaller.

3. We can construct OMLs with an arbitrary finite dimension of the space of group-valued measures. It suffices to take the product of the OML of Theorem 3.4 with a suitable Boolean algebra.

\section{ADDED IN PROOF}

After the paper was submitted for publication, Hans Weber (Potenza, Italy) informed me that he obtained an alternative solution. His proof uses a completely different method and computer proving.

\section{ACKNOWLEDGEMENT}

The author thanks B. Legan (Ruston) for a certain improvement in the original version of this paper, $\mathbf{H}$. Weber (Potenza) for e-mail communication leading to Corollary 2.2, and the members of the Prague quantum logic seminarP. Pták, V. Rogalewicz, and M. Matoušek-for their helpful comments.

\section{REFERENCES}

1. J. K. Brooks and R. S. Jewett, On finitely additive vector measures, Proc. Nat. Acad. Sci. U.S.A. 67 (1970), 1296-1298.

2. A. B. d'Andrea and P. de Lucia, The Brooks-Jewett theorem on an orthomodular lattice, J. Math. Anal. Appl. 156 (1991), 507-522.

3. P. de Lucia and P. Morales, A non-commutative version of a theorem of Marczewski for submeasures, Studia Math. (to appear).

4. P. de Lucia and T. Traynor, Non-commutative group-valued measures on an orthomodular poset (to appear).

5. R. J. Greechie, Orthomodular lattices admitting no states, J. Combin. Theory Ser. A. 10 (1971), 119-132.

6. R. J. Greechie and F. R. Miller, On structures related to states on an empirical logic I. Weights on finite spaces, Technical Report 16, Dept. of Math., Kansas State Univ., Manhattan, KA, 1970.

7. S. P. Gudder, Stochastic methods in quantum mechanics, North-Holland, New York, 1979.

8. J. Hamhalter and P. Pták, Hilbert-space-valued states on quantum logics, Appl. Math., vol. 37, Springer-Verlag, New York, 1992, pp. 51-61.

9. G. Kalmbach, Orthomodular lattices, Academic Press, London, 1983.

10. A. G. Kurosh., Group theory, Nauka, Moskow, 1967. (Russian)

11. M. Navara, P. Pták, and V. Rogalewicz, Enlargements of quantum logics, Pacific J. Math. 135 (1988), 361-369.

12. M. Navara and V. Rogalewicz, Construction of orthomodular lattices with given state spaces, Demonstratio Math. 21 (1988), 681-693.

13. _ State isomorphism of orthomodular posets and hypergraphs, Proc. 1st Winter School on Measure Theory, Liptovský, Ján, 1988, pp. 93-98. 
14. M. Navara, and G. T. Rüttimann, $A$ characterization of $\sigma$-state spaces of orthomodular lattices, Exposition. Math. 9 (1991), 275-286.

15. P. Pták, Exotic logics, Colloq. Math. 56 (1987), 1-7.

16. P. Pták and S. Pulmannová, Orthomodular structures as quantum logics, Kluwer, Dordrecht, Boston, and London, 1991.

17. F. W. Shultz, A characterization of state spaces of orthomodular lattices, J. Combin. Theory Ser. A 17 (1976), 317-328.

18. V. S. Varadarajan, Geometry of quantum theory. I, Van Nostrand, Princeton, NJ, 1968.

19. H. Weber, Compactness in spaces of group-valued contents, the Vitali-Hahn-Saks theorem and Nikodym's boundedness theorem, Rocky Mountain J. Math. 16 (1986), 253-275.

Department of Mathematics, Faculty of Electrical Engineering, Technical University of Prague, Technická 2, 16627 Prague, Czech Republic

E-mail address: navaramath.feld.crut.cz 